Annuaire suisse de politique de développement

23-2 | 2004

Les ONG de développement : rôles et perspectives

\title{
ONG et gouvernance mondiale : une légitimité contestée
}

Peter Niggli et André Rothenbühler

\section{(2) OpenEdition}

1 Journals

Édition électronique

URL : http://journals.openedition.org/aspd/447

DOI : 10.4000/aspd.447

ISSN : 1663-9669

Éditeur

Institut de hautes études internationales et du développement

Édition imprimée

Date de publication : 1 novembre 2004

Pagination : 25-31

ISSN : 1660-5934

Référence électronique

Peter Niggli et André Rothenbühler, "ONG et gouvernance mondiale : une légitimité contestée »,

Annuaire suisse de politique de développement [En ligne], 23-2 | 2004, mis en ligne le 08 mars 2010

consulté le 07 septembre 2020. URL : http://journals.openedition.org/aspd/447 ; DOI : https://doi.org/ 10.4000/aspd.447

(c) The Graduate Institute I Geneva 


\title{
ONG et gouvernance mondiale: une légitimité contestée ${ }^{1}$
}

\author{
Peter Niggli* et André Rothenbühler**
}

$P$

our beaucoup de gouvernements, d'organisations internationales et de sociétés multinationales, associer à leurs activités des organisations non gouvernementales (ONG) est aujourd'hui un must. Mais les mêmes instances critiquent de plus en plus les ONG et mettent en doute leur légitimité. Elles les voudraient bien un peu plus conciliantes - tout en manifestant un certain esprit de contradiction.

Depuis les premières manifestations contre des conférences intergouvernementales, les mondialistes accusent les ONG de s'arroger le rôle de gardiens des mœurs en matière de politique internationale. Il est, selon eux, absurde de qualifier les décisions intergouvernementales de non démocratiques, puisque les gouvernements ainsi vilipendés sont légitimés par des élections. Alors que les «permanents des $\mathrm{ONG}$ » ne représentent personne, et surtout pas la société civile.

Ce reproche de non-légitimité marque depuis lors le débat public sur les ONG, avec des appels non dissimulés à leur imposer une forme ou une autre de réglementation. Deux initiatives concrètes ont été lancées à ce sujet.

\section{Plaidoyers pour ou contre l'influence exercée par les ONG}

Kofi Annan, secrétaire général des Nations unies, a créé en été 2003 un Panel of Eminent Persons on Civil Society and UN Relationships présidé par Fernando Henrique Cardoso, ancien président du Brésil. Son objectif: «renforcer la présence de la société civile» dans la politique internationale et surtout au sein de l'ONU. Ce panel devait en outre présenter une série de «lignes directrices juridiques» auxquelles toutes les ONG seraient tenues de se référer. Mais il fallait aussi reconnaître que les ONG ont besoin d'une «certaine liberté » pour travailler - selon les termes de Kevin Kennedy, coordinateur du panel. Celui-ci partait de l'idée que même «non élues», les ONG ont le droit de représenter la société civile pour autant qu'elles respectent les règles des Nations unies.

Le rapport final du panel, remis à Kofi Annan en juin 2004, a transposé les questions délicates. Il préconise d'associer davantage les ONG au travail des instances onusiennes, de simplifier et d'unifier leur accréditation, et d'inclure dans le processus davantage d'ONG de l'hémisphère Sud. Ce rapport fait tout ce

1 Ce texte est une mise à jour d'un article paru dans Global + en décembre 2003.

* Directeur de la Communauté de travail Swissaid/Action de carême/Pain pour le prochain/Helvetas/ Caritas/EPER et responsable du secteur politique de développement. Sur la Communauté de travail, voir l'encadré en fin d'article.

** Collaborateur à la Déclaration de Berne et journaliste à l'agence de presse Infosud. 
qu'il peut pour calmer les inquiétudes manifestées à ce sujet par les gouvernements des pays en développement. Par contre, il n'aborde pas le sujet des «lignes directrices juridiques» censées servir de références à toutes les ONG.

D'autre part, au cours de l'été 2003, l'American Enterprise Institute, laboratoire d'idées du gouvernement Bush, a lancé avec des institutions apparentées le programme NGOWatch. Selon cet «observatoire», beaucoup d'ONG se sont écartées de leur mandat originel et se comportent comme des instances gouvernementales: la croissance extraordinaire d'ONG revendicatrices pourrait miner la souveraineté des démocraties constitutionnelles; les ONG donneraient la préférence à un ordre mondial basé sur la gouvernance globale (ou avec un gouvernement mondial), sapant ainsi la souveraineté des Etats nationaux démocratiques. Et l'ONU les soutiendrait dans cette voie. NGOWatch entend avertir le gouvernement américain et les multinationales des dangers qu'il y a à collaborer avec les ONG, et exiger de celles-ci davantage de transparence. Les ONG devraient cesser d'exercer des pressions politiques et revenir à leur mission caritative.

A en juger par son site Internet, NGOWatch n'a pas vraiment décollé. L'idée de profiter de la grande victoire en Irak pour tenter une mise au pas générale sur le front des ONG a perdu son élan avec les difficultés que devait affronter l'administration Bush face à l'échéance électorale. Et ce projet n'a pas encore eu un rayonnement appréciable sur le plan international non plus.

\section{«Enregistrement officiel » pour les ONG}

Le fait que les gouvernements doivent être élus pour agir au nom de leur pays constitue une exigence fondamentale. Mais cela ne signifie pas qu'il faille aussi élire les ONG pour que celles-ci puissent s'adresser à des gouvernements et les critiquer. Leur activité et leurs blâmes trouvent une légitimation suffisante dans les droits fondamentaux que comporte nécessairement un régime démocratique. C'est à l'opinion publique de décider si les critiques et les propositions des ONG valent quelque chose. Et même si le courant majoritaire de l'opinion rejetait les conceptions politiques des ONG, cela ne changerait rien au droit qu'ont celles-ci d'agir comme elles l'entendent. Tout le monde sait bien que les droits fondamentaux de la démocratie n'ont de réalité que si les idées minoritaires bénéficient du respect de la majorité et de la protection de la loi.

D'autre part, n'importe quelle multinationale s'attribue le droit de dialoguer en direct avec les gouvernements. Les lobbyistes de l'économie suivent depuis des décennies chaque négociation internationale et exercent leur influence sur la matière et le calendrier des tractations. D'importants accords gouvernementaux, à l'échelon régional ou international, résultent d'initiatives prises par des groupes ou des associations économiques, et ont largement tenu compte de leurs souhaits: c'est le European Roundtable of Industrialists (ERT) qui a lancé l'idée et le projet du Marché intérieur de l'UE, et l'association des entreprises américaines du secteur tertiaire a fait de même pour l'Accord général sur le commerce des services $(\mathrm{AGCS})^{2}$ de l'Organisation mondiale du commerce (OMC). Pourtant, ces groupes d'entreprises et ces associations économiques agissent ainsi sans être élus ou mandatés par leur personnel ou par la population de leurs pays d'origine.

2 En anglais GATS, General Agreement on Trade in Services. 
Certaines ONG contribuent toutefois à entretenir la confusion du débat en défendant leur légitimité sans se référer à la notion des droits fondamentaux. Elles affirment «représenter» la société civile et prétendent assumer ainsi une fonction que n'a pratiquement aucune ONG - mis à part les syndicats ou les organisations paysannes. Avec une telle ambition, elles n'ont bien entendu guère d'arguments pour réfuter le reproche que personne ne les a chargées de représenter la société civile. A notre avis, la raison d'être de nombreuses ONG est non pas de représenter qui que ce soit, mais de rappeler l'existence de groupes et de problèmes qui ne parviennent pas à faire valoir leurs points de vue et qui risquent d'être occultés par l'affrontement des intérêts dominants.

Les milieux critiques à l'égard des ONG n'abordent pas - pour des raisons évidentes - la question des droits politiques fondamentaux. Ils préconisent en lieu et place de nouveaux instruments pour combler le prétendu déficit de légitimité. C'est ainsi que le Foreign Policy Centre, laboratoire d'idées du gouvernement britannique, a proposé des règles de comportement pour les ONG ainsi que la création d'une autorité de surveillance chargée de leur certification. Ces propositions visent à légitimer les ONG par une reconnaissance officielle, tout en instaurant des règles destinées à canaliser leur fonction critique. Cela donnerait aussi aux autorités le moyen de départager les $\mathrm{ONG}$ «raisonnables» des «extrémistes».

\section{Problème de légitimité ou de gestion?}

Certaines voix s'élèvent contre l'absurdité de ce débat sur la légitimité des ONG. Le New York Times, par exemple, a commenté ainsi l'initiative NGOWatch: "Il va de soi que l'on peut discuter et critiquer librement les opinions politiques des $\mathrm{ONG}$; mais l'obligation de rendre compte et la transparence devraient se rapporter à leur gestion, et non pas à leur politique. »

Ce journal rappelle ainsi que les ONG sont aussi des entreprises fournissant des services, vivant de dons et souvent bénéficiaires de subsides des pouvoirs publics. Il est normal d'exiger que leur gestion fasse preuve de transparence et se soumette à l'obligation de rendre compte. Comme la plupart sont exonérées d'impôts, leur manière de gérer leurs affaires doit de toute manière obéir à des règles imposées par l'Etat. Dans beaucoup de pays dont la Suisse, les ONG se soumettent en outre volontairement à des normes destinées à assurer que les dons servent bien à l'affectation prévue. Actuellement, de nouvelles initiatives voient le jour ici et là pour renforcer la gouvernance d'entreprise des $\mathrm{ONG}$, ce qui témoigne de la professionnalisation de la branche et de sa croissance.

Souvent, on ne sait pas très bien si la ligne de conduite évoquée pour les ONG dans le cadre du débat sur leur légitimité se rapporte à leur comportement politique ou à des règles de «bonne gouvernance». Il est bien entendu justifié d'exiger une gestion correcte, mais s'il s'agit de bonne conduite politique, cela devient discutable.

\section{Déficit démocratique de la politique internationale}

Ce débat sur la légitimité des ONG émane à l'origine de certains membres de gouvernements des pays industrialisés et des multinationales, sérieusement agacés par le mouvement antimondialiste. Ces milieux souhaitent couper entièrement de 
cette mouvance les principales ONG spécialisées dans l'environnement, le développement ou les droits de l'homme, et qui jouissent d'un grand crédit dans l'opinion. Mais les mêmes instances cherchent également à systématiser la collaboration avec ces $\mathrm{ONG}$, dans l'espoir de mieux légitimer leurs actions intergouvernementales.

Lorsqu'ils négocient des accords et mettent sur pied des institutions internationales, les gouvernements exercent un double, et parfois un triple pouvoir - législatif, exécutif et judiciaire. Il n'y a pas ici les checks and balances (système de contrepoids) qui caractérisent un Etat démocratique. Les résultats de ces négociations violent en outre les législations nationales dans le domaine économique, sans que les citoyens, les groupes d'intérêts et le parlement du pays considéré n'aient été vraiment consultés. Le fait que les gouvernements soient élus sur le plan national ne suffit pas à légitimer leur abondante activité législative à l'échelle internationale. Ce déficit démocratique de la politique internationale a contribué dans une mesure décisive à la naissance du mouvement antimondialisation.

Lorsque les gouvernements autorisent des ONG à suivre les conférences internationales, leur communiquent certaines informations triées sur le volet ou les consultent sur des affaires en cours, cela contribue à masquer leur déficit démocratique. Si des ONG vont même jusqu'à critiquer les gouvernements et revendiquer d'autres solutions devant l' «opinion mondiale», cela renforce l'apparence de débat démocratique. Quand ensuite un gouvernement affirme à une de ses commissions parlementaires qu'il a consulté les ONG à propos des affaires internationales importantes, il suggère avoir pris en considération autant que possible tous les points de vue de l'opposition. Ce qui, bien entendu, n'est généralement pas le cas. On voit donc que les ONG critiques sont non seulement importunes - raison pour leurs adversaires de mettre en doute leur légitimité -, mais aussi utiles.

\section{Un statut de «tiers état» pour les ONG?}

L'ONU prône depuis deux ou trois ans, avec l'appui des gouvernements des pays industrialisés, le principe des coopérations tripartites entre gouvernements, société civile et milieux économiques. Le panel Cardoso a pris pour hypothèse qu'il y a trois types d'acteurs internationaux - à savoir gouvernements, société civile et secteur privé -, dont il s'agit d'orchestrer les interactions et qui détermineront la future gouvernance globale. On se dirigerait ainsi vers un régime corporatiste dans lequel les ONG feraient en quelque sorte office de «tiers état».

Michael Edwards, directeur de la Governance and Civil Society Unit de la Ford Foundation, une importante source de financement d'ONG américaines et internationales, a approfondi dans un livre l'idée du modèle corporatiste. A son avis, il faudrait «structurer» le droit qu'ont les ONG de se mêler des affaires publiques par une série de compacts (accords) entre gouvernements, entreprises et réseaux d'ONG - et cela de bas en haut, donc de l'échelon régional à la dimension internationale en passant par l'étape nationale. Ces accords régleraient «les rôles et les responsabilités » des acteurs concernant certains thèmes et à l'égard de certaines institutions. Edwards estime que son modèle a pour avantage qu'il empêche les ONG de passer outre à l'échelon national (donc l'entente politique à ce niveau) pour porter les conflits directement sur la scène internationale. Il donne comme exemple le projet hydroélectrique Arun III au 
Népal, que la Banque mondiale voulait cofinancer et qui a échoué en raison d'une campagne internationale lancée par des ONG: on aurait simplement passé par-dessus le gros travail de coalition building mené à l'échelon national, et prêté trop peu d'attention aux défenseurs locaux de ce projet.

Le modèle d'Edwards profiterait aux puissants et affaiblirait l'influence des ONG. Il met d'autre part en évidence les problèmes que comporte l'approche corporatiste pour la gouvernance globale: si l'on applique un tel système à l'échelon national, il y aura immédiatement conflit avec les mécanismes démocratiques existants. Imaginons un accord pour la Suisse entre Conseil fédéral, Economiesuisse et les ONG - quel sera l'accueil des parlements aux trois niveaux, des exécutifs cantonaux et communaux, des partis politiques et des institutions de la démocratie directe? Si l'accord prévaut, il provoquera un soulèvement des Suissesses et des Suisses; et son assujettissement aux règles démocratiques lui ferait par contre perdre tout sens. L'économie aussi bien que les ONG s'en tirent mieux sur le plan national en faisant simplement usage des mécanismes démocratiques, sans recourir à un concept corporatiste. Il n'y a aucune raison pour que ce modèle fonctionne mieux et soit plus démocratique dans les pays en développement. Le corporatisme tripartite n'est envisageable que sur le plan international ou dans des Etats autoritaires, où la démocratie est absente.

En résumé, les ONG ont tout lieu de ne pas s'abandonner inconsidérément à la tentation d'une gouvernance globale structurée dans un esprit corporatiste. Il vaut naturellement mieux que les ONG soient associées aux questions de gouvernance globale et n'abandonnent pas le terrain aux représentants des grands groupes économiques, lesquels ont de toute manière l'habitude d'intervenir depuis longtemps sur la scène internationale. A notre avis, c'est là le principal argument que les ONG devraient avancer pour justifier leur revendication d'être consultées en matière de gouvernance globale. Mais cela ne suffira encore pas pour démocratiser la politique internationale. Il faut donc que les ONG continuent de critiquer le déficit démocratique de la gouvernance globale et qu'elles restent particulièrement sceptiques à l'égard de la tendance à transférer des compétences politiques du niveau national au niveau international.

\section{SOURCES}

Edwards Michael, «NGOs and International Economic Policy-Making: Rights and Responsibilities in the Global Arena», World Economics, vol. 2, no 3, July-September 2001, pp. 127-137.

—, NGO Rights and Responsibilities: A New Deal for Global Governance, London, Foreign Policy Centre, 2000.

NGOWatch, <www.NGOWatch.org $>$.

United Nations, We the Peoples: Civil Society, the United Nations and Global Governance, Report of the Panel of Eminent Persons on United Nations-Civil Society Relations, juin 2004, <www.un.org/ reform/panel.htm>. 


\section{La Communauté de travail: une mission de lobbying et de plaidoyer}

La Communauté de travail des organisations d'entraide (CdT) relève des six grandes organisations suisses qui la financent. Deux d'entre elles sont associées au catholicisme suisse (Action de carême et Caritas), deux aux Eglises protestantes (Pain pour le prochain et EPER), les deux dernières étant neutres sur le plan idéologique et religieux (Swissaid et Helvetas). La CdT a été fondée en 1971 par Swissaid, Pain pour le prochain et Action de carême. Ses autres membres s'y sont joints plus tard, le dernier étant I'EPER en 2003.

La Communauté de travail n'est pas une association faîtière et n'exerce aucune fonction de coordination pour ses membres. Ceux-ci ont confié à l'organisme qu'ils ont créé sous le nom de "Communauté de travail " une mission de lobbying et de plaidoyer auprès de l'opinion publique, sur des thèmes choisis de politique de développement.

\section{Champs d'action}

\section{Coopération et politique de développement}

1. Théorie et pratique, ainsi que conflits d'objectifs, de la coopération au développement et de l'aide humanitaire suisses.

2. Conflits d'objectifs découlant des interventions politiques de différentes instances gouvernementales en relation avec les pays du Sud (problèmes de cohérence).

3. Théorie et pratique de la coopération multilatérale au développement, notamment politique suisse au sein de la Banque mondiale et dans d'autres organismes analogues.

4. Politique internationale et politique suisse en matière de dettes et de désendettement.

\section{Politique économique internationale}

5. Politique financière internationale, politique suisse au sein du Fonds monétaire international (FMI) et aspects de la place financière suisse ayant des incidences politiques sur le développement.

6. Politique commerciale internationale, politique économique extérieure de la Suisse et politique suisse au sein de I'Organisation mondiale du commerce (OMC).

7. Comportement des multinationales suisses dans les pays en développement, politique internationale et Nord-Sud de leurs associations nationales et internationales.

\section{Gouvernance globale}

8. Politique environnementale internationale et position de la Suisse dans ce contexte.

9. Autres problèmes de gouvernance globale (ONU, sommets extraordinaires de I'ONU, G-7, politique mondiale des Etats-Unis et d'autres grandes puissances) en relation avec la politique de développement, et politique suisse à cet égard.

\section{Objectifs poursuivis}

1. Elle entend obtenir que le Parlement corrige ou atténue la décision prise par le Conseil fédéral de financer la contribution suisse à la cohésion de l'UE sur le budget du développement. Le Conseil fédéral a résolu en 2004 d'imputer entièrement cette contribution - totalisant 1 milliard de francs et répartie sur cinq ans - aux Ministères de l'économie et des affaires étrangères; I'un et l'autre s'exécuteront aux dépens de la coopération au développement. Cela aurait ainsi pour conséquence que les bénéficiaires de l'aide au développement - les pays les plus pauvres - feraient les frais des bilatérales II (entre autres la protection de l'évasion fiscale) sans participer aux avantages que la Suisse tire de ses nouveaux accords avec I'UE. Même certains milieux conservateurs du Parlement estiment la méthode assez choquante. Si l'on ne corrige pas cette décision, I'aide au développement suisse s'en trouverait réduite de quelque $20 \%$ alors que le Conseil fédéral continue de prétendre accroître cette aide de manière à ce qu'elle atteigne 0,4\% du produit national brut en 2010 .

2. Le système international de fraude fiscale que pratiquent les acteurs les plus mobiles de l'économie mondiale porte préjudice à tous les Etats et prive les pays en développement de quelque 50 milliards de dollars par année; les organisations multilatérales doivent s'en occuper et prendre des mesures éner- 
giques pour y mettre un frein. II faut que la Suisse assouplisse et supprime au cours des dix ans à venir sa protection officielle des fraudeurs étrangers, trompeusement appelée secret bancaire. La Communauté de travail a mis sur pied à cet effet un réseau international d'ONG intitulé Tax Justice Network, afin de combattre les méthodes d'extraterritorialité pratiquées par l'Angleterre, la France, la Belgique ou la Suisse, en essayant d'amener l'OCDE, I'ONU et d'autres organisations internationales concernées à instaurer une répression coordonnée de ces pratiques.

3. Il convient de remplacer les négociations "globales» de I'Organisation mondiale du commerce (OMC) par une série de négociations spécifiquement destinées à corriger le caractère unilatéralement défavorable aux pays en développement des accords existants, en adoptant de nouvelles règles qui doivent permettre à ces pays de mettre à nouveau en place une politique économique capable de protéger leurs intérêts. II s'agit d'amener le Conseil fédéral à approuver des négociations dans ce sens et à encourager les pays industrialisés dans la voie de concessions unilatérales, sans contre-prestations "équivalentes». Depuis Seattle, la CdT a passé une alliance informelle avec les syndicats, toutes les associations paysannes, les grandes organisations écologistes et les organisations de développement pour observer la politique suivie par le Conseil fédéral à l'égard de I'OMC. Cette alliance regroupe tous les milieux dont les intérêts ou les préoccupations morales seraient mis en péril par la nouvelle poussée de libéralisation commerciale que les pays industrialisés cherchent à imposer depuis Seattle (1999).

4. L'accès à l'eau potable est un droit humain qu'il s'agit de codifier par une convention internationale. La CdT a créé dans ce but un réseau international d'ONG qui s'emploie à inciter des pays appropriés à prendre l'initiative d'une telle convention. Elle croyait au départ que la Suisse allait s'associer activement à ce projet, le Parlement ayant transmis il y a trois ans un postulat dans ce sens au Conseil fédéral. Celui-ci a promis alors de s'engager pour que cette convention voie le jour. L'affaire s'est ensablée entre-temps dans l'administration. Des fonctionnaires font de la résistance parce qu'ils nient l'urgence d'une convention et craignent que cela fasse obstacle à la privatisation de l'approvisionnement en eau dans le Sud. Ils pensent que seuls des investissements massifs du secteur privé assureront l'eau potable pour tout le monde, oubliant que les milieux privés investissent plus volontiers dans un système d'approvisionnement en eau si des subsides et des garanties de l'Etat couvrent entièrement leurs risques. Afin de progresser, la CdT a pris cette année l'initiative d'un réseau international de grandes ONG; en font partie notamment l'organisation allemande Brot für die Welt, le Council of Canadians, WeDo (réseau international Femmes et développement). Ces Friends of a Convention diffusent l'idée d'une convention sur l'eau et cherchent à collaborer avec des Etats, des organisations onusiennes et d'autres institutions intéressées. La CdT reprendra contact avec le gouvernement suisse lorsque ceux d'autres pays seront devenus favorables à une telle convention. 